\title{
Identification of the typical miRNAs and target genes in hepatocellular carcinoma
}

\author{
KEQIANG HAN，JING LI，HONGZHI ZHAO，PING LIANG，XIAOBING HUANG, \\ LU ZHENG, YUMING LI, TONGHAN YANG and LIANG WANG
}

Department of Hepatobiliary Surgery, Xinqiao Hospital, The Third Military Medical University, Chongqing 400037, P.R. China

Received June 25, 2013; Accepted February 6, 2014

DOI: $10.3892 / \mathrm{mmr} .2014 .2194$

\begin{abstract}
The aim of the present study was to identify miRNAs that were differentially expressed in hepatocellular carcinoma (HCC) by comparing normal and cancer tissue samples and to analyze the correlation of the target genes and HCC. The gene expression profile of GSE31383 was downloaded from the Gene Expression Omnibus database, including 19 samples, 9 normal and 10 from HCC tissue samples. The differentially-expressed miRNAs were identified with packages in $\mathrm{R}$ language and further analyzed using bioinformatics methods. Firstly, the verified targets of miRNAs were integrated in two miRNA databases: miRecords and miRTarBase, and the targets of the differentially-expressed miRNAs were obtained. The software STRING was then used to construct the interaction network of target genes. Finally, a functional enrichment analysis of the genes in the interaction network was conducted using the software Gestalt. Typical miR-224 and miR-214 were identified by comparing normal and cancer samples, each of which obtained 14 and 8 target genes, respectively. The functional enrichment analysis of the targets in the two groups highlighted the intracellular signaling cascade. In conclusion, the featured miRNAs (the upregulated miRNA-224 and downregulated miRNA-214) and their target genes are significant in the occurrence and development of HCC, which is likely to be significant for the identification of therapeutic targets and biomarkers to aid in the treatment of HCC.
\end{abstract}

\section{Introduction}

Hepatocellular carcinoma ( $\mathrm{HCC}$, also termed malignant hepatoma) is one of the most common types of cancer and it is the second most common cause of cancer-related mortality in

Correspondence to: $\mathrm{Dr}$ Xiaobing Huang, Department of Hepatobiliary Surgery, Xinqiao Hospital, The Third Military Medical University, 183 Xinqiao Avenue, Chongqing 400037, P.R. China

E-mail: xiaobinghuang@hotmail.com

Key words: hepatocellular carcinoma, differentially-expressed miRNA, target gene, interaction network, function enrichment analysis
China. Common with other cancers, $\mathrm{HCC}$ is a complex disease that develops when a mutation of the cellular machinery occurs, which results in the cell replicating at a higher rate or avoiding apoptosis (1). Due to the complicated pathophysiology of HCC, it is difficult to treat this cancer successfully and the prognosis is unsatisfactory. For example, only 10-20\% of HCCs can be completely removed by surgery, which may lead to recurrence of this disease within 3-6 months. However, the molecular mechanisms involved in this biological process remain to be fully understood. Thus further studies are required and may contribute to the identification of key factors in HCC progression.

Molecular changes in HCC are complex, including the dysregulated genes, protein markers, non-coding RNAs and other predictive biomarkers. Numerous dysregulated genes and potential protein markers in HCC have been reported in the past decades. For instance, the prospero homeobox protein (2) and high-mobility group A2 (3) were identified as critical factors that regulate transcription factors relevant to epithelial-mesenchymal transition, a significant event during tumorigenesis, to promote HCC metastasis. Key dysregulated genes involved in $\mathrm{HCC}$ have been identified by genomic and gene expression analysis, including the oncogenes c-myc and N-ras and the tumor suppressor genes tumor protein 53, retinoblastoma protein $1(R b 1)$, cyclin-dependent kinase inhibitor 2A $(C D K N 2 A)$ and Axin 1. These genes were located on the regions of the chromosome of genomic and epigenetic changes that were linked with HCC development (4).

There is recent evidence indicating that microRNAs (miRNAs; a type of non-coding RNA) have significant roles in cancer, in particular in cancer development, progression and metastasis, and as robust biomarkers for cancer prognosis. For example, miR-129-2 methylation is highly accurate in distinguishing HCC from cirrhosis patients and healthy individuals, implying its potential utility as an early diagnostic marker for HCC (5). MiR-20a (6) and miR-195 (7) exhibit significant inhibitory roles in $\mathrm{HCC}$ progression and may be potential therapeutic targets and biomarkers for patients with HCC. It is noteworthy that certain miRNAs have been revealed to target multiple genes involved in HCC progression, including miR-221 and miR-222 that can target $p 27, p 57, \mathrm{~B}$-cell lymphoma-2 (Bcl2)-modifying factor, phosphatase and tensin homolog, metalloproteinase inhibitor 3, DNA-damage-inducible transcript 4 protein and protein phosphatase 2 , regulatory 
subunit $\mathrm{B} \alpha$; and miR-122 can target cyclin G1, disintegrin and metalloproteinase domain (ADAM)-containing protein 10, $A D A M 17$, serum response factor, insulin-like growth factor 1 receptor and Bcl-w (8). These findings imply potential utility of miRNAs as therapeutic targets and biomarkers for different pathophysiological processes of HCC. Thus it is necessary to provide an overview of miRNA expression and determine their target genes. In addition, the development of computational prediction to identify potential miRNA targets is convenient for the functional investigation of miRNAs (9).

The present study aimed to identify featured target genes of significant differentially-expressed miRNAs of HCC by comparing normal and cancer tissue samples, and to analyze the correlation of the target genes and HCC. Candidate target genes identified by these approaches may provide the groundwork for a combination therapy approach for HCC. However, further investigation of their potential use in the treatment of $\mathrm{HCC}$ is required.

\section{Materials and methods}

Microarray data. The miRNA profiling data was obtained from the study by Wang et al (10), which was found in the GEO (Gene Expression Omnibus) database (ID: GSE31383) (11) and included nine human healthy liver and $10 \mathrm{HCC}$ samples. The miRNA profiling was performed successfully using freshly frozen healthy liver and HCC tissues obtained at the time of surgical treatment. The annotation information of a total of 19 chips was available based on the GPL10122 Platform (version 4; Luminex, New York, NY, USA) that was designed by the bead-based technology.

Data pre-process and differentially-expressed miRNA analysis. The microarray data in CEL format files were converted into expression measures with the affy package (Bioconductor) (12) in R language $(13,14)$ and standardized with the median method. Next, the LIMMA package (Bioconductor) in R (15) was used to identify the miRNAs that were differentially expressed between the nine human healthy liver and the 10 HCC samples. Subsequently, the consequences were adjusted for multiple testing with the Benjamin and Hochberg (16) method in a multi-test package. The miRNAs only with false discovery rate $($ FDR $)<0.05$ and llogFold Changel $>1$ were selected, and the differentially-expressed miRNA that showed the greatest level of upregulation (or downregulated) was selected for further analysis.

Screening the target genes of the differentially-expressed $m i R N A$. In order to obtain the accurate target genes, the two selected miRNAs were integrated into two miRNA databases: miRecords (http://mirecords.biolead.org) (17) and miRTarBase (http://mirtarbase.mbc.nctu.edu.tw/) (18), in which the confirmed target genes had been selected as functional genes of miRNAs.

The miRTarBase, a comprehensive database, contains a manually curated collection of $>1,300$ experimentally supported miRNA targets in several animal species, plants and viruses. The miRecords, an integrated resource of interactions of experimentally validated miRNA targets, consists of two components, the Validated Targets component and the Predicated Targets

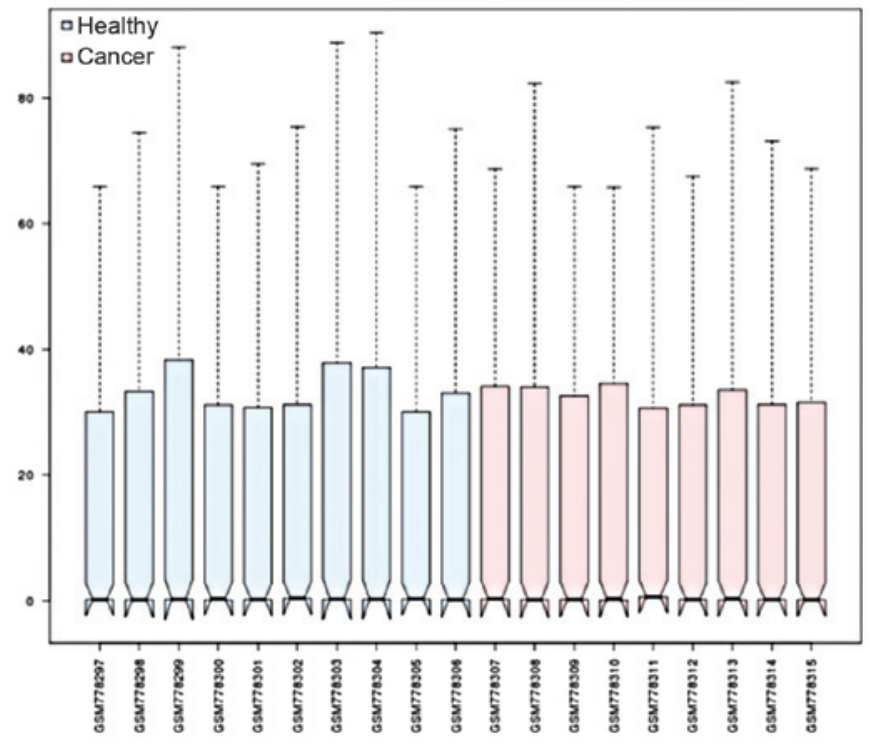

Figure 1. The cassette figure of the standardization expression value. The blue boxes represent normal samples and the pink boxes represent cancer tissues. Black lines show the median of each data and its distribution represents the standardization degree of the data. The black lines in figure are almost on the same straight line, which suggests that their expression value is at the same level.

component, and hosts 2,286 records of interactions between 548 miRNAs and 1,579 target genes in nine animal species.

Network construction. The Search Tool for the Retrieval of the Interacting Genes (STRING) database (19) was used to search and construct the interaction network of the target genes of the differentially-expressed miRNAs. Each score represented a rough estimate of how likely a given association describes a functional linkage between two genes. The pairs with a combination score $>0.5$ were recorded. Finally, the interaction network was analyzed, and the degree of each node was calculated according to the experimental database and data mining of the software.

Functional enrichment analysis. Functional enrichment analysis of the genes in the interaction network was performed with software Gestalt (Leuven, Belgium; Gene Set Analysis Toolkit) (20), an integrated system for analyzing gene sets in various biological contexts, which incorporates information of eight species, including humans, rats and mice, and different public resources, including NCBI (21), Ensemble (22), KEGG (23) and GO (24). The function of algorithm based on the hypergeometric distribution (25) and pathway enrichment analysis (26) in this software identified the correlated genes in the interaction network. FDR $<0.05$ was set as the cut-off criterion.

\section{Results}

Screening differentially-expressed miRNAs. To identify the specific differentially-expressed miRNAs between human HCC tissues and healthy controls, publicly available microarray datasets GSE31383 were obtained from the GEO database. The miRNA expression profiling data were preprocessed with the $\mathrm{R}$ affy package and were normalized by the median method (Fig. 1). 
Table I. Differentially expressed miRNAs $(\mathrm{FDR}<0.05, \mid \log \mathrm{FCl}>1)$.

\begin{tabular}{|c|c|c|c|}
\hline miRNA name & ID & FDR & $\log \mathrm{FC}$ \\
\hline hsa-miR-214 & EAM249 & $1.24 \times 10^{-5}$ & -6.85536 \\
\hline hsa-miR-503 & JLA186 & $6.19 \times 10^{-11}$ & -6.53656 \\
\hline hsa-mir-378 & JLA120 & $9.35 \times 10^{-5}$ & -6.05122 \\
\hline hsa-miR-200b & EAM305 & $1.20 \times 10^{-3}$ & -5.9841 \\
\hline hsa-miR-424 & JLA103 & $7.07 \times 10^{-4}$ & -4.3832 \\
\hline hsa-miR-375 & JLA106 & $2.54 \times 10^{-3}$ & -4.2444 \\
\hline hsa-miR-497 & JLA147 & $2.02 \times 10^{-2}$ & -4.02889 \\
\hline hsa-mir-30a & EAM280 & $6.36 \times 10^{-3}$ & -3.75307 \\
\hline hsa-miR-200c & JLA216 & $2.02 \times 10^{-2}$ & -3.69177 \\
\hline hsa-miR-130a & EAM159 & $6.36 \times 10^{-3}$ & -3.26364 \\
\hline hsa-miR-99a & EAM121 & $3.84 \times 10^{-2}$ & -2.88995 \\
\hline hsa-miR-145 & EAM212 & $1.44 \times 10^{-3}$ & -2.75653 \\
\hline hsa-miR-361-5p & JLA105 & $4.89 \times 10^{-2}$ & -2.28546 \\
\hline hsa-miR-451 & JLA5 & $3.69 \times 10^{-2}$ & -2.15525 \\
\hline hsa-miR-335 & EAM385 & $2.02 \times 10^{-2}$ & -2.08815 \\
\hline hsa-miR-101 & EAM311 & $1.90 \times 10^{-2}$ & -1.69783 \\
\hline hsa-miR-195 & EAM299 & $3.50 \times 10^{-5}$ & -1.65589 \\
\hline hsa-let-7c & EAM145 & $3.84 \times 10^{-2}$ & -1.14039 \\
\hline hsa-miR-146a & EAM139 & $1.54 \times 10^{-2}$ & 1.38159 \\
\hline hsa-miR-21 & EAM244 & $1.20 \times 10^{-4}$ & 1.7009 \\
\hline hsa-miR-7 & EAM109 & $2.81 \times 10^{-2}$ & 2.55763 \\
\hline hsa-miR-524-5p & JLA170 & $3.84 \times 10^{-2}$ & 2.6693 \\
\hline hsa-miR-10b & EAM190 & $7.07 \times 10^{-4}$ & 3.39984 \\
\hline hsa-miR-222 & EAM258 & $2.58 \times 10^{-2}$ & 4.12295 \\
\hline hsa-miR-520g & JLA176 & $3.69 \times 10^{-2}$ & 4.81125 \\
\hline hsa-mir-520c & JLA17 & $1.90 \times 10^{-2}$ & 5.26842 \\
\hline hsa-mir-519d & JLA29 & $2.02 \times 10^{-2}$ & 5.50376 \\
\hline hsa-miR-517c & JLA180 & $2.98 \times 10^{-2}$ & 5.63958 \\
\hline hsa-miR-516b & JLA27 & $2.02 \times 10^{-2}$ & 5.72554 \\
\hline hsa-miR-155 & EAM317 & $2.77 \times 10^{-3}$ & 6.19272 \\
\hline hsa-miR-517a & JLA26 & $2.76 \times 10^{-2}$ & 6.72391 \\
\hline hsa-miR-224 & EAM323 & $2.96 \times 10^{-5}$ & 6.87628 \\
\hline
\end{tabular}

miRNA, microRNA; FDR, false discovery rate; ID, identification.

At an $\mathrm{FDR}<0.05$ and $\mid \log \mathrm{FCl}>1$, a total of 32 miRNAs was considered to be differentially-expressed between the healthy controls and human HCC tissues, including 18 downregulated and 14 upregulated miRNAs (Table I). miRNA-224, exhibited the greatest upregulation and thus, was selected from the 18 upregulated miRNAs. Similarly, miRNA-214 was selected from the downregulated group (Fig. 2).

By integrating the verified targets of miRNAs downloaded from miRecords and miRTarBase, 14 target genes of miRNA-224 and 8 target genes of miRNA-214 were obtained (Table II), respectively.

Interaction network construction of target genes. The target genes of miRNA-224 and miRNA-214 were mapped using the software STRING and to predict their interactions. By integrating these correlations, interaction networks between the target genes and their interactive genes were constructed (Fig. 3). The core of the interaction network of miRNA-224 targets (Fig. 3A) included CD40 [combined with CD40 ligand (LG), score 0.999], cell division cycle 42 (CDC42; combined with Wiskott-Aldrich syndrome-like, score 0.999), chemokine (C-X-C motif) receptor (CXCR) 4 (combined with protein kinase $\mathrm{C}, \zeta$ (PRKCZ; score 0.913), and platelet-derived growth factor receptor, $\beta$ polypeptide (PDGFRB; combined with phosphoinositide-3-kinase, regulatory subunit 2, score 0.999) (27-29). However, the network of miRNA-214 targets and their interactive genes (Fig. 3B) revealed that mitogen-activated protein kinase (MAPK) 8 was in the core and program organized unit (POU) domain, class 4, transcription factor 2 (POU4F2) only interacted with cyclin D1 (CCND1).

Functional enrichment analysis of the interaction network. In total, 15 and 13 function-clusters from the two interaction 
Table II. Target genes of the two significantly differential expressed miRNAs.

A, hsa-miR-224

\begin{tabular}{lll}
\hline Target gene name & Source & \\
\hline AP2M1 & miRTarBase & Test method \\
& miRecord & \\
miRTarBase & Luciferase reporter assay \\
API-5 & miRecord & Western blotting//Luciferase reporter assay//qPCR \\
& miRTarBase & activity assay// RT-PCR \\
CD40 & miRTarBase & Microarray//qPCR \\
CDC42 & miRTarBase & Luciferase reporter assay//qPCR//Western blotting \\
CXCR4 & miRTarBase & Luciferase reporter assay//qPCR//Western blotting \\
EDNRA & miRTarBase & Northern blot//qPCR//Western blotting \\
EYA4 & miRTarBase & Northern blot//qPCR//Western blotting \\
FOSB & miRTarBase & Luciferase reporter assay \\
KLK10 & miRecord & qPCR//Luciferase reporter assay \\
& miRTarBase & Luciferase activity assay// Western blotting \\
NCOA6 & miRTarBase & Luciferase reporter assay \\
NIT1 & miRTarBase & Luciferase reporter assay \\
PDGFRB & miRTarBase & Microarray//Northern blotting \\
RAB9B & miRecord & Microarray//Northern blotting \\
KLK1 & ELISA
\end{tabular}

B, hsa-miR-214

\begin{tabular}{lll}
\hline Target gene name & Source & \\
\hline DAPK1 & miRTarBase & Test method \\
EZH2 & miRTarBase & Microarray//qPCR \\
MAP2K3 & miRTarBase & Luciferase reporter assay//Northern blot \\
MAPK8 & miRTarBase & GFP reporter assay//Microarray//qPCR//Western blot \\
PLXNB1 & miRTarBase & GFP reporter assay//Microarray//qPCR//Western blot \\
POU4F2 & miRTarBase & Immunohistochemistry//qPCR//Western blot \\
PTEN & miRTarBase & GFP reporter assay//Northern blot//qPCR//Western blot \\
& miRecord & Western blot//qPCR//Luciferase reporter assay \\
SRGAP1 & miRTarBase & RT-PCR \\
& & Luciferase reporter assay//qPCR//western blot
\end{tabular}

AP2M1, adaptor-related protein complex 2, $\mu 1$ subunit; API-5, apoptosis inhibitor 5; CDC42, cell division cycle 42; CXCR4, chemokine (C-X-C motif) receptor 4; EDNRA, endothelin receptor type A; EYA4, eyes absent homolog 4; FOSB, FBJ osteosarcoma oncogene B; KLK, kallikrein-related peptidase; NCOA6, nuclear receptor coactivator 6; NIT1, nitrilase 1; PDGFRB, platelet-derived growth factor receptor, beta polypeptide; DAPK1, death-associated protein kinase 1; EZH2, enhancer of zeste homolog 2; MAPK, mitogen-activated protein kinase; PLXNB1, plexin B1; PTEN, phosphatase and tensin homolog; SRGAP1, SLIT-ROBO Rho GTPase activating protein 1; qPCR, qunatitative polymerase chain reaction; RT-PCR, reverse transcription PCR.

networks were gained via the functional enrichment analysis with software Gestalt (Fig. 4). For the miRNA-224 targets, the most significantly enriched function-cluster of the total 15 categories was the cell signaling cascade and others included phosphorus and phosphate metabolic processes and the regulation of apoptosis.

As for the 13 categories of the miRNA-214 targets, in which were in accordance with the 13 functional-clusters of the 15 categories in miRNA-224 targets, the interactive gene number of each functional-cluster in miRNA-214 targets was larger than that in miRNA-224 targets. In the common
13 functional-clusters in miRNA-214 and in miRNA-224, the interactive genes of each target were all correlated with the intracellular signaling cascade (Fig. 4, circled in red).

\section{Discussion}

HCC, one of the most common types of tumor, results in 662,000 mortalities per year worldwide and the prognosis of $\mathrm{HCC}$ is poor (30). Therefore, there is an urgent requirement to investigate the mechanism of HCC and to develop an effective preventative strategy. Recently, in a hotspot study of HCC, 

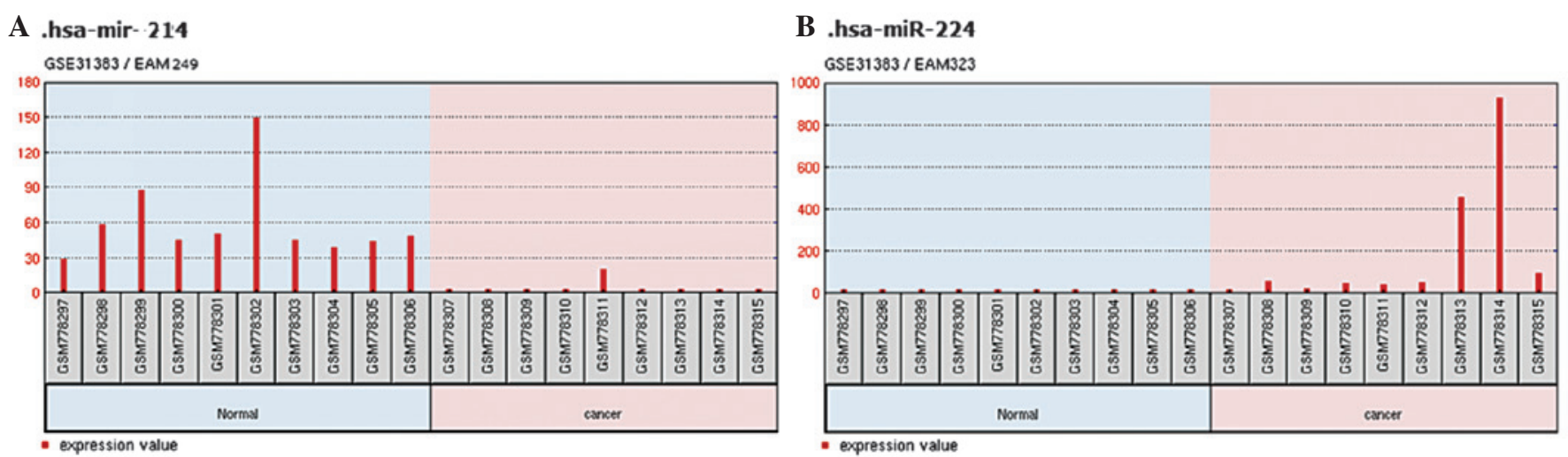

Figure 2. Expression-level of two selected micro (mi)RNAs. (A) Expression value of the downregulated miRNA-214. (B) Expression value of the upregulated miRNA-224.
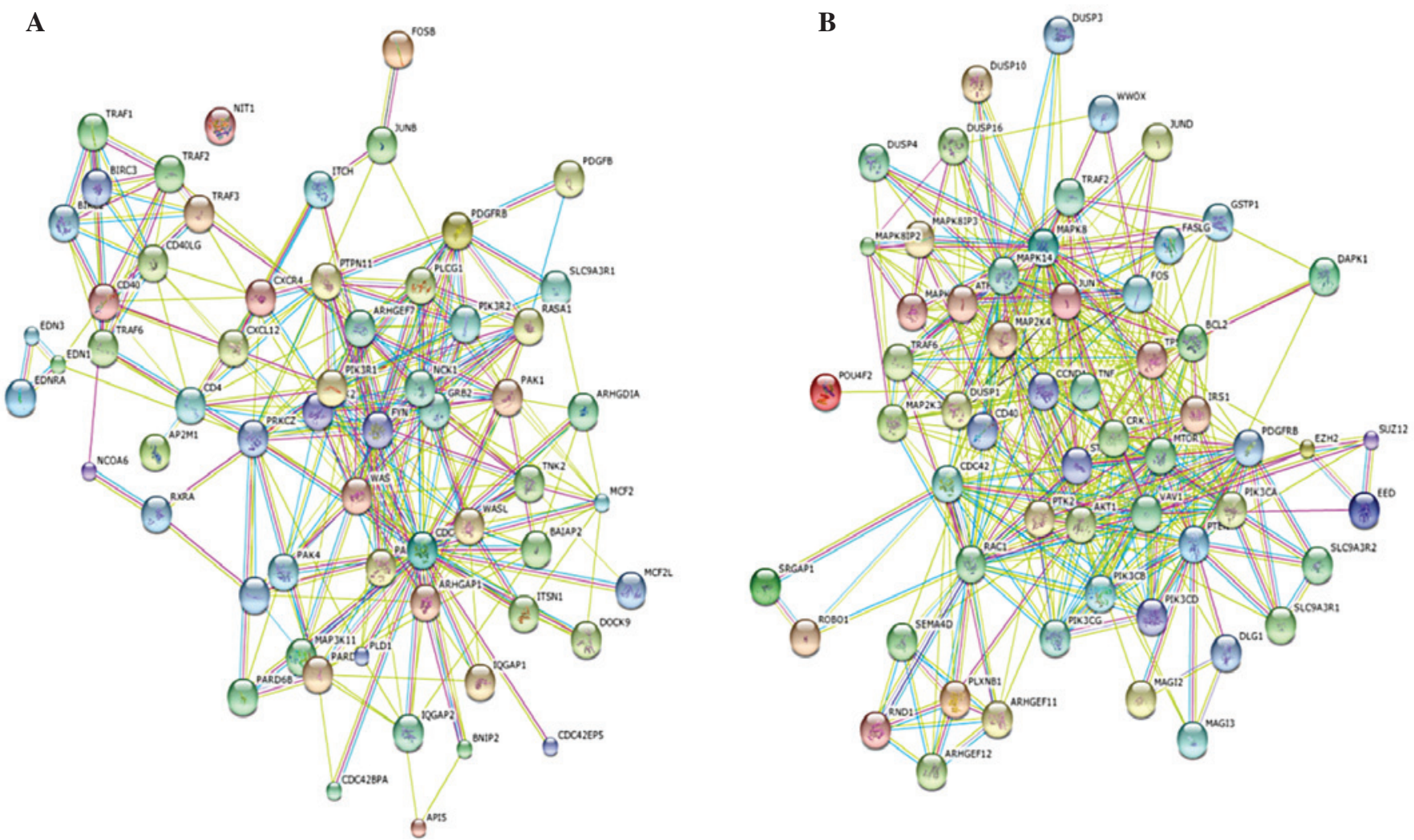

Figure 3. Interaction networks of target genes constructed by Search Tool for the Retrieval of the Interacting Genes. (A) Interaction networks of target genes of miRNA-224. (B) Interaction networks of target genes of miRNA-214.

it has been demonstrated that miRNAs may represent novel potential therapeutic targets and biomarkers for patients with $\operatorname{HCC}(31,32)$.

In the present study, the gene expression profile GSE31383 from GEO was used to analyze the differentially-expressed miRNAs between HCC and healthy samples (10). As a result, two featured miRNAs were obtained, the upregulated miRNA-224 and downregulated miRNA-214, which were significantly differentially-expressed, as well as 22 target genes, 14 targets of miRNA-224 and eight targets of miRNA-214. These results were concurrent with a number of previous studies, which demonstrated the important role of miR-224 and its targets in cell proliferation, migration, invasion and anti-apoptosis of $\mathrm{HCC}$ by directly binding to interactive genes (33). Target CD40 was demonstrated to be the only upregulated molecule in the presence of hepatitis B virus. CD40LG was the major signal that induced $B$ cells to efficiently present antigen to T cells. The CD40-CD40LG interaction was crucial in the activation of antigen-presenting cells and in the initiation of humoral and cellular immune responses (34). Studies demonstrated that CD40LG-mediated immune gene therapy for HCC was an effective treatment as it activated the humoral and cellular immune systems $(35,36)$. In addition, PRKCZ has been proposed to induce hepatocyte growth factor to regulate the CXCR4-CXC ligand 12, which has been demonstrated to 


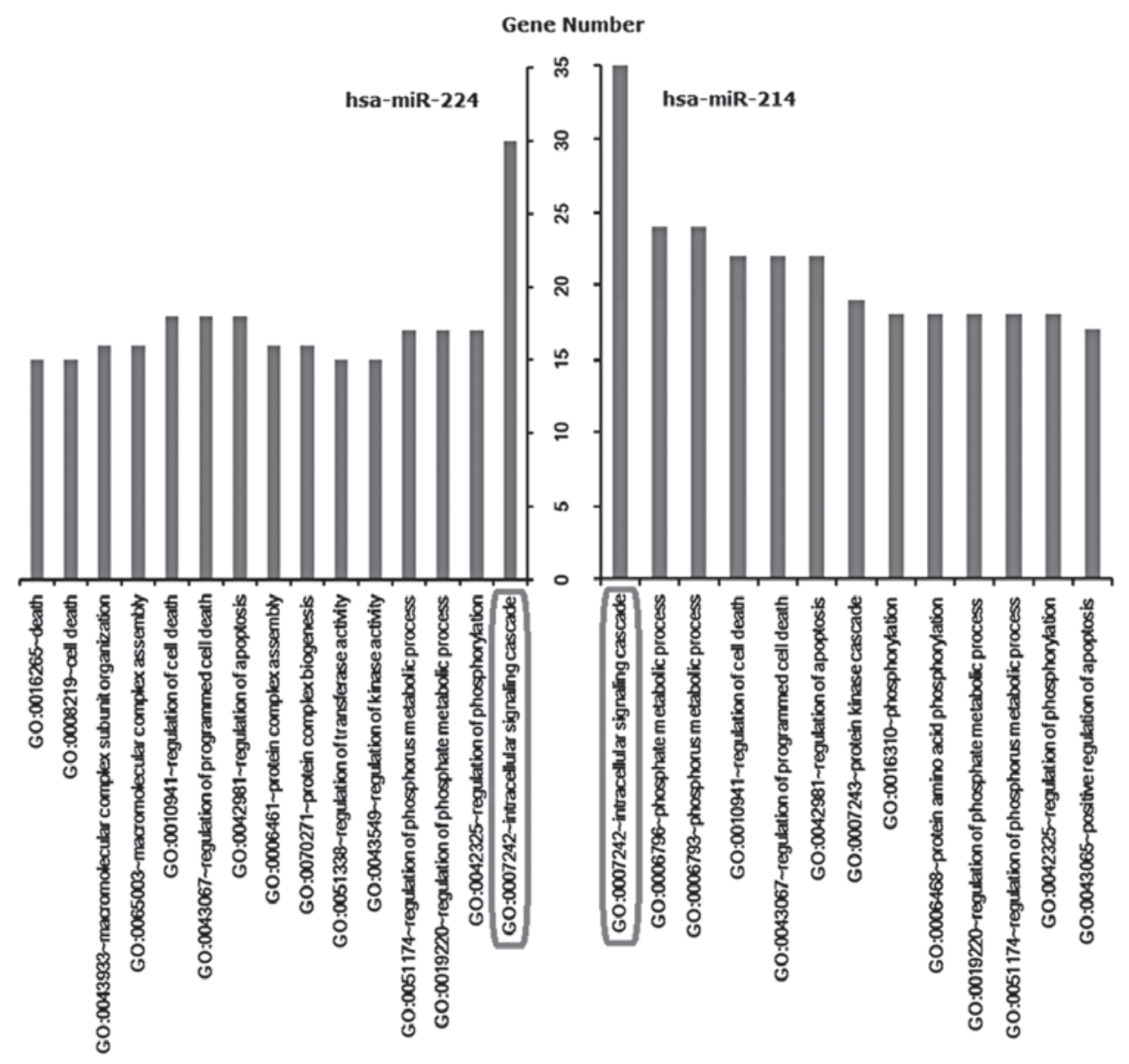

Figure 4. Column chart of the functional enrichment analysis of the target genes in each interaction networks.

mediate the metastasis of a number of malignant tumors (37). In the network analysis of the present study, it was initially identified that PRKCZ directly combined with the target gene CXCR4 in HCC, which requires further analysis and experimental verification (38). However, it was noteworthy that the target gene nitrilase (NIT) 1 did not combine with any other gene. Further functional analysis of NIT1 is required and may be useful for the clarification of HCC development.

miR-214 is likely to act as a tumor suppressor and to have significant roles in inhibiting tumorigenesis of HCC by suppressing the growth factors, such as EZH2, CTNNB1 and $\mathrm{CDH} 1$, in human hepatoma (39). In the network of eight target genes of miR-214, the majority of targets were not in the core region and a number of interactive genes also appeared in the network of miR-224. The target gene POU4F2 has been demonstrated to function as a transcriptional factor in breast cancer (40); however, no study has been reported with regard to the interaction of POU4F2 and CCND1.

Finally, the functional enrichment analysis of interactive genes of the two target groups were enriched to 15 and 13 function-clusters respectively. In each cluster, the most significant function was the intracellular signaling cascade. The initiation of intracellular signaling cascades may occur via cell-substratum interactions, triggering subcellular events, as reported previously (41). The interactive genes correlated with this function included the tumor necrosis factor (TNF) receptor-associated factor, phosphatidylinositol 3-kinase
(PI3K) and MAPK, which had been demonstrated to function in the regulation of inflammation, antiviral responses and apoptosis of HCC $(42,43)$. Clinical trials of target genes combined with inhibitors, such as p16 and p27, have been conducted for decades and have revealed positive outcomes for treating HCC (44). Researchers reported that activation of PI3K/AKT and MAPK pathway through a PDGFRB-dependent feedback loop compromised the antitumor activity of rapamycin in HCC, and blockade of this feedback loop by sorafenib was an attractive approach to improve the antitumor effect of rapamycin, particularly in preventing or treating $\mathrm{HCC}$ recurrence following liver transplantation (27). However, the present study confirmed anticancer effects of a synthesized novel PI3K $\alpha$ inhibitor on HCC cells and the inhibitor exhibited anticancer activities (45). These results demonstrated the potential function of miRNAs and their targets in the treatment of HCC.

In conclusion, miR-224 and miR-214 were identified by comparing normal and HCC tissue samples and the targeted genes regulated by miRNAs and their interaction network indicated that they were correlated with the intracellular signaling cascade, a significant pathway of signaling transduction. The target genes and their associated interactive genes, are significant in cellular responses, including proliferation regulation, gene expression, differentiation, mitosis, cell survival and apoptosis, and are likely to be presented as novel potential therapeutic targets and biomarkers of HCC. Further analyses are required to unravel the involvement HCC development. 


\section{Acknowledgements}

This study was supported by the Natural Science Foundation Project of CQ CSTC (grant no. cstc2011jjA10001).

\section{References}

1. Chen CJ, Yang HI, Su J, et al: Risk of hepatocellular carcinoma across a biological gradient of serum hepatitis B virus DNA level. JAMA 295: 65-73, 2006.

2. Liu Y, Zhang JB, Qin Y, et al: PROX1 promotes hepatocellular carcinoma metastasis by way of up-regulating hypoxia-inducible factor $1 \alpha$ expression and protein stability. Hepatology 58 692-705, 2013.

3. Luo Y, Li W and Liao H: HMGA2 induces epithelial-to-mesenchymal transition in human hepatocellular carcinoma cells Oncol Lett 5: 1353-1356, 2013

4. Zhao H, Wang J, Han Y, et al: ARID2: a new tumor suppressor gene in hepatocellular carcinoma. Oncotarget 2: 886-891, 2011.

5. Lu CY, Lin KY, Tien MT, Wu CT, Uen YH and Tseng TL: Frequent DNA methylation of MiR-129-2 and its potential clinical implication in hepatocellular carcinoma. Genes Chromosomes Cancer 52: 636-643, 2013.

6. Shrivastava S, Petrone J, Steele R, Lauer GM, Bisceglie AM and Ray RB: Up-regulation of circulating miR-20a is correlated with hepatitis $\mathrm{C}$ virus-mediated liver disease progression. Hepatology 58: 863-871, 2013.

7. Ding J, Huang S, Wang Y, et al: Genome-wide screening revealed that miR-195 targets the TNF- $\alpha / N F-\kappa B$ pathway by downregulating IкB kinase alpha and TAB3 in hepatocellular carcinoma. Hepatology 58: 654-666, 2013.

8. Huang $\mathrm{S}$ and $\mathrm{He} \mathrm{X}$ : The role of microRNAs in liver cancer progression. Br J Cancer 104: 235-240, 2011.

9. Barbato C, Arisi I, Frizzo ME, Brandi R, Da Sacco L and Masotti A: Computational challenges in miRNA target predictions: to be or not to be a true target? J Biomed Biotechnol 2009: 803069, 2009.

10. Wang PR, Xu M, Toffanin S, Li Y, Llovet JM and Russell DW: Induction of hepatocellular carcinoma by in vivo gene targeting. Proc Natl Acad Sci USA 109: 11264-11269, 2012.

11. Barrett T, Troup DB, Wilhite SE, et al: NCBI GEO: mining tens of millions of expression profiles - database and tools update. Nucleic Acids Res 35 (Suppl 1): D760-D765, 2007.

12. Gautier L, Irizarry R, Cope L and Bolstad B: Description of Affy. http://www.bioconductor.org/packages/release/bioc/ vignettes/affy/inst/doc/affy.pdf. Accessed April 15, 2014.

13. Fujita A, Sato JR, Rodrigues Lde O, Ferreira CE and Sogayar MC: Evaluating different methods of microarray data normalization. BMC Bioinformatics 7: 469, 2006.

14. Troyanskaya O, Cantor M, Sherlock G, et al: Missing value estimation methods for DNA microarrays. Bioinformatics 17 $520-525,2001$

15. Smyth GK: Limma: linear models for microarray data. In: Bioinformatics and Computational Biology Solutions using $\mathrm{R}$ and Bioconductor. Gentleman R, Carey V, Huber W, Irizarry R and Dudoit S (eds). Springer New York, NY, pp397-420, 2005.

16. Diboun I, Wernisch L, Orengo CA and Koltzenburg M: Microarray analysis after RNA amplification can detect pronounced differences in gene expression using limma. BMC Genomics 7: 252, 2006.

17. Xiao F, Zuo Z, Cai G, Kang S, Gao X and Li T: miRecords: an integrated resource for microRNA-target interactions. Nucleic Acids Res 37: D105-D110, 2009.

18. Hsu SD, Lin FM, Wu WY, et al: miRTarBase: a database curates experimentally validated microRNA-target interactions. Nucleic Acids Res 39: D163-D169, 2011.

19. Szklarczyk D, Franceschini A,Kuhn M, et al: The STRING database in 2011: functional interaction networks of proteins, globally integrated and scored. Nucleic Acids Res 39: D561-D568, 2011.

20. Zhang B, Kirov S and Snoddy J: WebGestalt: an integrated system for exploring gene sets in various biological contexts. Nucleic Acids Res 33: W741-W748, 2005.

21. Edgar R, Domrachev M and Lash AE: Gene Expression Omnibus: NCBI gene expression and hybridization array data repository. Nucleic Acids Res 30: 207-210, 2002.
22. Zwanzig R: Ensemble method in the theory of irreversibility. J Chem Phys 33: 1338, 1960.

23. Kanehisa M and Goto S: KEGG: kyoto encyclopedia of genes and genomes. Nucleic Acids Res 28: 27-30, 2000.

24. Hulsegge I, Kommadath A and Smits MA: Globaltest and GOEAST: two different approaches for Gene Ontology analysis. BMC Proc 3 (Suppl 4): S10, 2009.

25. Exton H (ed): Handbook of Hypergeometric Integrals: Theory, Applications, Tables, Computer Programs. Ellis Horwood, Chichester, England, 1978.

26. Weng L, Macciardi F, Subramanian A, et al: SNP-based pathway enrichment analysis for genome-wide association studies. BMC Bioinformatics 12: 99, 2011.

27. Li QL, Gu FM, Wang Z, et al: Activation of PI3K/AKT and MAPK pathway through a PDGFR $\beta$-dependent feedback loop is involved in rapamycin resistance in hepatocellular carcinoma. PLoS One 7: e33379, 2012.

28. Stengel $\mathrm{K}$ and Zheng Y: Cdc42 in oncogenic transformation, invasion, and tumorigenesis. Cell Signal 23: 1415-1423, 2011.

29. Manu KA, Shanmugam MK, Ong TH, et al: Emodin suppresses migration and invasion through the modulation of CXCR4 expression in an orthotopic model of human hepatocellular carcinoma. PLoS One 8: e57015, 2013.

30. Shi YH, Ding ZB, Zhou J, et al: Prognostic significance of Beclin 1-dependent apoptotic activity in hepatocellular carcinoma. Autophagy 5: 380-382, 2009.

31. Llovet JM and Bruix J: Molecular targeted therapies in hepatocellular carcinoma. Hepatology 48: 1312-1327, 2008.

32. Zhu AX, Sahani DV, Duda DG, et al: Efficacy, safety, and potential biomarkers of sunitinib monotherapy in advanced hepatocellular carcinoma: a phase II study. J Clin Oncol 27: 3027-3035, 2009.

33. Zhang Y, Takahashi S, Tasaka A, Yoshima T, Ochi $\mathrm{H}$ and Chayama K: Involvement of microRNA-224 in cell proliferation, migration, invasion, and anti-apoptosis in hepatocellular carcinoma. J Gastroenterol Hepatol 28: 565-575, 2013.

34. Jiang YF, Ma J, He Y, Zhang YH, Xu Y and Gong GZ: Cationic liposome-mediated transfection of CD40 ligand gene inhibits hepatic tumor growth of hepatocellular carcinoma in mice. J Zhejiang Univ Sci B 10: 7-13, 2009.

35. Tang Y, Chen Y, Ni B, Yang D, Guo S and Wu Y: Up-regulation of the expression of costimulatory molecule CD40 in hepatocytes by hepatitis B virus X antigen. Biochem Biophys Res Commun 384: 12-17, 2009.

36. Wan Y, Ma X, Li X and Yi J: A novel immunotherapy to hepatocellular carcinoma: CD40-activated B lymphocytes transfected with AFPmRNA. Med Hypotheses 73: 835-837, 2009.

37. Huang S, Ouyang N, Lin L, et al: HGF-induced PKC $\zeta$ activation increases functional CXCR4 expression in human breast cancer cells. PLoS One 7: e29124, 2012.

38. Liu Y, Wang B, Wang J, et al: Down-regulation of PKCzeta expression inhibits chemotaxis signal transduction in human lung cancer cells. Lung Cancer 63: 210-218, 2009.

39. Xia H, Ooi LL and Hui KM: MiR-214 targets $\beta$-catenin pathway to suppress invasion, stem-like traits and recurrence of human hepatocellular carcinoma. PLoS One 7: e44206, 2012.

40. Fujita R, Ounzain S, Wang AC, Heads RJ and Budhram-Mahadeo VS: Hsp-27 induction requires POU4F2/Brn-3b TF in doxorubicin-treated breast cancer cells, whereas phosphorylation alters its cellular localisation following drug treatment. Cell Stress Chaperones 16: 427-439, 2011.

41. Golemis EA and Chernoff J: Analysis and manipulation of intracellular signaling cascades. Methods 32: 347-348, 2004.

42. Zhang L, Jiang G, Yao F, et al: Growth inhibition and apoptosis induced by osthole, a natural coumarin, in hepatocellular carcinoma. PLoS One 7: e37865, 2012.

43. Zhang DM, Liu JS, Deng LJ, et al: Arenobufagin, a natural bufadienolide from toad venom, induces apoptosis and autophagy in human hepatocellular carcinoma cells through inhibition of PI3K/Akt/mTOR pathway. Carcinogenesis 34: 1331-1342, 2013.

44. Matsuda Y, Wakai T, Kubota M, et al: Clinical significance of cell cycle inhibitors in hepatocellular carcinoma. Med Mol Morphol 46: 185-192, 2013.

45. Yun SM, Lee JH, Jung KH, et al: Induction of apoptosis and suppression of angiogenesis of hepatocellular carcinoma by HS-159, a novel phosphatidylinositol 3-kinase inhibitor. Int J Oncol 43: 201-109, 2013. 